\title{
Social Groups as Objects: How Groups Can be Considered Objects within the Eliminativist Account
}

\author{
Chris Mayer ${ }^{1}$ \\ ${ }^{1}$ Ph.D., Lieutenant Colonel, U.S. Army, Associate Dean for Strategy, Policy, and Assessment, Academy Professor of \\ Philosophy, United States Military Academy, West Point, NY, USA. 845-938-3334 \\ Correspondence: Chris Mayer, Ph.D., Lieutenant Colonel, U.S. Army, Associate Dean for Strategy, Policy, and \\ Assessment, Academy Professor of Philosophy, United States Military Academy, West Point, NY, USA. \\ Disclaimer: The views expressed in this article are those of the author and do not reflect the position of the United \\ States Military Academy, the Department of the Army, or the Department of Defense.
}

Received: September 1, 2015

doi:10.11114/ijsss.v4i1.1203
Accepted: September 14, 2015

Available online: November 17, 2015

URL: http://dx.doi.org/10.11114/ijsss.v4i1.1203

\begin{abstract}
The power of groups over their members has been well documented in psychological, sociological, and political literature. There is, however, not as much written about the metaphysical status of these groups or the implications of this status. Trenton Merricks establishes a criterion for determining whether something counts as an object, and he concludes that only persons meet this criterion. While at first this designation seems trivial, it does have implications for how we treat and view those things we view as objects and for how we treat and view non-objects. In this article, I argue that some groups meet the criterion proposed by Merricks for distinguishing objects from non-objects by establishing characteristics these groups must meet to be considered objects. If I am right, these groups then deserve the status and rights that come with being a person.
\end{abstract}

Keywords: groups, metaphysics, loyalty, beliefs

\section{Introduction}

In Objects and Persons, Trenton Merricks, in describing his view of eliminativism, claims that for something to be an object it must possess non-redundant causal power. What we think of as statues are not objects, while persons are objects because their mental causation provides non-redundant downward causal control over their parts. The fundamental tenets of Merricks' argument, as well as his view that persons are objects, seem plausible. However, Merricks focuses exclusively on persons and excludes groups of persons as possible. I think this is an omission because certain types of groups of persons can be considered objects, even if one accepts the eliminativism that Merricks proposes. In what follows I will argue that some groups of persons can be considered objects because their properties do not supervene on their parts (their members); these groups possess non-redundant downward causal control over the persons that compose them.

\section{The Eliminativist View}

In this section I will summarize the basic tenets of Merricks eliminativist viewpoint. These ideas will be important as I employ them later to argue that some groups should be considered objects.

As I mentioned above, Merricks (2003) proposes a view of eliminativism that denies that statues exist. Instead, what we think of as statues are simply atoms arranged statuewise. This view is further specified in the following statement:

Atoms are arranged statuewise if and only if they both have the properties and also stand in the relations to microscopica upon which, if statues existed, those atoms composing a statue would non-trivially supervene. (p. 4)

An initially puzzling idea is that, although Merricks denies that statues exist, eliminativism relies upon the concept of statue. Yet the use of this concept does not undermine the eliminativist view. Merricks (2003) accepts that "the folk concept of statue plays a role in determining which atomic arrangements are statuewise." (p. 8) Eliminativists employ folk concepts in order to show that what we think of as statues are really atoms arranged statuewise. According to Merricks, to argue that eliminitavism is inconsistent because it employs the concept of statue is misguided. The use of the folk concept of statue in the eliminativist proposal in no way implies that statues exist. Moreover, as Merricks (2003) 
notes, the use of folk concepts in everyday life is neither deceptive (because the eliminativist refers to an object that she knows does not exist) nor is it a repudiation of eliminativism. It is simply recognition of the concepts we use to communicate. The relevance of the use of folk concepts to this paper is that people, or even the eliminativist, could also make reference to the concept of groups without having to accept the claim that groups exist.

One benefit of the eliminativist view is that it avoids the problem of causal overdetermination. According to Merricks (2003), overdetermination of an effect occurs when:

1. "that effect is caused by an object";

2. "that object is causally irrelevant to whether some other-i.e numerically distinct-object $\quad$ or objects cause that effect";

3. "the other object or objects do indeed cause that effect." (p. 58)

To illustrate the problem of overdetermination, Merricks employs the example of a baseball breaking a window. The breaking of the window can be explained solely by the atoms arranged baseballwise; no reference to the baseball as an object is needed to explain why the window shattered (Merricks, 2003). Additionally, "the shattering of the window is not overdetermined" (Merricks, 2003, p. 56) Only one cause is needed to explain the shattering of the window. Thus, atoms arranged baseballwise do the work to shatter the window; the (alleged) baseball does nothing. Or, as Merricks (2003) states, "everything (alleged) baseballs and other non-living macrophysical objects (allegedly) cause is caused by their proper parts at some level of decomposition" (p. 80). Given this, things we often think of as objects, such as baseballs, are not in fact objects. To be considered objects they would have to possess "non-redundant downward causal control over their constituent atoms" (Merricks, 2003, p. 84). That is, to be an object something must have the power to intentionally cause its atoms to do something. It is clear that baseballs do not have this sort of causal power, but do humans?

I will not go into all of the arguments that Merricks provides in support of the view that humans are objects; many will come out as I provide my argument as to why some groups can be considered objects. Merricks' basic idea is that human consciousness, through mental properties, allows for downward causal control over the atoms that compose a human. When humans perform an action, we cannot explain the action solely by referring to our atomic level; the action occurs because humans have non-redundant causal power.

Merricks provides three points that support his claim that persons can be thought of as objects. The first is that we have a special "epistemic access to our own existence"; we can look in the mirror and determine that the human organism that is reflected in the mirror exists and has causal power. Second, if you eliminate persons, and call them atoms arranged humanwise, you would be claiming that there are "thinkings and speakings but neither thinkers nor speakers." Eliminating persons eliminates the causes of thoughts and the spoken word; a person as a thinker and speaker is the best explanation for the existence of thoughts and the spoken word. Finally, you can explain everything that a baseball causes without the baseball; you cannot do the same for things caused by a person (Merricks, 2003). These three reasons provide support for Merricks' contention that human organisms, given the criteria he proposes, are objects. These types of reasons will be essential for determining whether groups are objects as well.

\section{Merricks' View on Groups}

Before I present the characteristics of social groups, I want to explore some of Merricks' thoughts on groups. Merricks does not directly address whether groups are objects; however, he does make reference to groups in his defense of eliminativism. The examples he provides are useful because they present possible problems for the view that groups are objects. In one passage Merricks (2003) writes:

There is a crew aboard ship is true of any fully manned ship (pretend there are ships), if it means only that there are many people aboard, performing certain assigned tasks. But 'there is a crew aboard ship' is false if it means that those people stand in the relation of composing something one to another. It is false, that is, if it means that on the ship there is a big scattered object composed of those people, an object with a mass many times that of any person and a constantly shifting and hard-to-locate center of gravity. (p. 15)

In the discussion that follows this passage, Merricks does not take a definite position on groups, but instead uses this example to make a point against the linguistic charge. But, this passage does highlight some problems associated with thinking of groups as objects. Although we refer to the crew as an object, we may not really mean that it is one. This is because if the crew were an object, there would be no other object like it (assuming that things like chairs were objects). The crew's parts (its members) are scatted all over the ship, and it is hard to pin down its exact location at a given time.

A similar point can be made using Merricks' discussion of water in the pool. Many would consider the water an object because we can see it as one mass (Merricks, 2003). Merricks asks the reader to instead consider whether what we think 
of as the water is simply $\mathrm{H}_{2} \mathrm{O}$ molecules. Since $\mathrm{H}_{2} \mathrm{O}$ molecules in the pool seem to have the same effects as those we typically grant to water in the pool, nothing is lost by considering the water to actually be a collection of molecules rather than as a whole object. Claiming that the water in the pool is an object would imply overdetermination. This type of objection could possibly apply to groups: claiming that groups exist would imply overdetermination if everything that a group caused could be explained by what its members cause.

\section{Defining Social Groups}

If I am to show that groups are objects, I will need to show how a group meets the eliminativist criteria for an object and avoids the likely eliminativist criticism. First, though, I must lay out what I take a group to be. I will use the term social group to distinguish groups that are objects from those that are not.

A social group is more than just a collection of two or more people with similar properties; the people must be related to each other in a certain way. This means that "the population of left-handed people, or the population of women, is not automatically a social group by virtue of having members with a common property" (Gilbert, 1989, p. 9). To be considered a social group, a collection of people must meet certain conditions. Even large corporations may, as Margaret Gilbert suggests, lack the necessary characteristics of a social group. In these corporations there is "a Kafkaesque sense of disconnection in everyone's mind" (Gilbert, 1989, p. 231). The organization's members do not consider the organization's purpose as their own, and they do not share a sense of unity with the other members. In what follows I will specify three conditions that social groups must meet to be considered an object under Merricks' view of eliminativism.

\subsection{Joint Commitment}

Margaret Gilbert and Paul Sheehy disagree on the type of commitment required for a collection of people to be considered a social group. Gilbert's view is grounded in intentionality while Sheehy's is not. Gilbert requires that each group member freely and openly commit him or herself to the group's purpose. Members must also know that other members have done the same, and all members must recognize that a group exists and think of themselves as part of it (Gilbert, 1989). Sheehy (2006) presents the intentionality thesis as the view that "individuals can constitute a social group only when each believes or understands himself to be linked in some salient way with the others or when each conceives of himself as a member of the group" (p. 58). Although Sheehy (2006) admits that this is a commonly accepted view, he criticizes Gilbert for assuming intentionalism without providing an adequate argument for it (p. 60).

Sheehy's counterexample to Gilbert's intentionalist view is the forming of a mob. Sheehy (2006) admits that the "mob is perhaps the most ephemeral of social groups," but argues that the mob has causal power by having some effect on its members' actions and on the world without meeting the intentionality condition prescribed by Gilbert (P. 77). Sheehy's mob example does provide the effects that Gilbert and others want (myself included) without the intentionalist baggage; however, the mob seems to be so unstable that it would come into and out of existence too quickly to be considered a social group. Additionally, the mob's members lack the strong relationship to one another that social groups require.

Joint commitment need not possess the strict intentionality and formality that Gilbert requires, but it does need to be more robust than Sheehy's version. Group participants do not necessarily need to "openly express something," as Gilbert (2003) claims; however it should be "common knowledge between the parties" that a commitment exists (p. 52). This common knowledge includes members recognizing that they have formed a group and that they are working towards the group's ends. In cases where the group is a formal organization, it is fairly clear when members have intentionally joined the group and have openly expressed their commitment. There can be cases, though, where this commitment is not openly expressed nor done intentionally, but still can be considered commitment.

One case of this might be a group of students who "hang-out" together in school. While they have not formally created a social group (in the way that a gang might be created), they are committed to each other by certain characteristics. Perhaps their socio-economic background is what ties them together. Thus, they dress similarly, have interest in the same things, eat lunch together, and spend time together on the weekends. ${ }^{1}$ Their commitment is expressed in their actions, and group members can recognize each other's commitment by observing these actions. ${ }^{2}$ These actions provide group members with the knowledge that they are committed to each other, even if this is not done intentionally. If one member of the group (Sally) starts dressing differently or eating lunch with a different group of people, other members may not be so sure that Sally is still committed to them. Her actions suggest that she may not be as committed as before.

It is important to note that a group may contain members who are not jointly committed to the group and its goals. This

\footnotetext{
1 I thank Brian Cwik for offering this example during my presentation.

${ }^{2}$ To avoid the problem associated with the mob, actions that express commitment must be performed over a significant length of time.
} 
fact, however, does not mean that a group with uncommitted members is not a social group. What is needed for a social group to exist, in terms of joint commitment, is a critical mass of committed individuals. It is hard to quantitatively identify this critical mass, but it seems essential that there are enough members to make it plausible that the group can achieve its goals and possess the other conditions that I discuss below. Joint commitment is also essential in providing group members with a sense of "we," when speaking of each other, and "it," when speaking of the group. This identity affirms the agency of the group and is an indication that the group is more than a collection of individuals who happen to be working together; it is a social group.

\subsection{Group Unity}

Sheehy (2006) proposes that "groups are composite material particulars formed through interrelating individuals" (p. 4). It is evident, however, that groups lack the type of material unity that is possessed by a human being. All parts of a human are connected together in a relatively small space. It is easy to see an entire human body, while it is often difficult to see an entire group because its members are typically not contained in one location and are not physically connected. Yet groups are composed of material objects (making them a material object), and their interrelation is what holds together the group. In The Process of Social Organizations, Marvin Olsen lays out three characteristics of groups necessary for group unity. One is that groups have established boundaries, which include people that serve as members; members' identification with each other and the group; and location of the group (Olsen, 1968, p. 67). Without these boundary conditions it is difficult to get an accurate picture of the group and what it consists of. These conditions provide the initial outline of the group, much as a person's silhouette and physical appearance would provide the initial impression of him or her.

Also important to establishing group unity is the structural stability that it possesses. "Its basic form and essential features can be differentiated from the social environment only if the overall structure of the organization persists for some minimal time" (Olsen, 1968, p. 69). The mob example that Sheehy presents is useful, but in the end it fails to meet this criterion of stability. The mob is too random and temporary to be considered a group, for it is typically the case that a mob lasts a short period of time and then dissipates without any chance of reforming. It would be odd to characterize something so unstable an object because its structure prevents long-term influence of its environment and its members.

The last characteristic of a group is a unique culture. This culture includes the group's goals, commitments, ideas, beliefs and values. The culture explains what the group is, what it seeks, and what it considers important (Olsen, 1968). It is analogous to the beliefs, ideas, and goals of a person that are essential in defining who we consider that person to be. Without a culture, a collection of individuals cannot be considered a group, for a culture is essential in providing direction for the group.

\subsection{Group Consciousness}

A final component of a social group is that it contains some sort of mental process that addresses the group's problems and guides its actions. Philip Pettit suggests that some groups are "minded [in a way] that is starkly discontinuous with the mentality of their members." In his view this group mind "is strong enough to ground talk of such collectives as entities that are psychologically autonomous and that constitute institutional persons" (Pettit, 2003, p. 167). What Pettit argues is not for a physical mind, but rather a rational process that is separate and distinct from any one member or even the entire membership. To illustrate this point, he presents numerous examples of problems confronted by a three person group. One example shows a three judge panel considering whether a defendant is liable. Two issues (whether a contract was in place and whether there was a breach in contract) must be decided prior to each judge rendering a decision on whether the defendant is liable. To illustrate the group thought process, Pettit presents the following matrix:

Table 1. Group Consciousness

\begin{tabular}{llll}
\hline Judge & Contract in Place? & Breach of Contract? & Liable? \\
\hline A & Yes & No & No \\
B & No & Yes & No \\
C & Yes & Yes & Yes
\end{tabular}

For the defendant to be found liable, there must be evidence that a contract was in place and that the defendant breached it. Only judge $\mathrm{C}$ can individually conclude that the defendant was liable (because she decided that a contract was in place and that it was breached). If we just consider the conclusions of each judge, the defendant is not found liable (Since judges A and B decided against liability). However, Pettit suggests that groups often reason by evaluating individual premises rather than summing up the judgments of each participant. In the case at hand, if we focus on the premises, we see that in both cases (whether a contract was in place and whether it was breached) a majority of the judges decided in the affirmative. Consequently, if the group's view is taken on each premise, the conclusion would be 
that the defendant is guilty (Pettit, 2003, p. 169). This demonstrates the existence of a group's thought process that is distinct from the thought process of its members.

A possible counterexample to Pettit's judge case is as follows. Suppose you have three rocks, each weighing one pound, and you put them on a scale together. The resulting weight, three pounds, is different than what the rocks weigh individually. Despite this difference, we would not consider the three rocks placed on the scale to be an object or to have created anything new as compared to the three one pound rocks considered separately. Does Pettit's case differ from the case of placing three rocks on a scale? ${ }^{3}$

To be analogous to the rock case, Pettit's case would have to take the individual final judgments of A, B, and C and simply sum them; this is done when the scale captures the sum of the three rocks. Doing this in Pettit's case provides a two to one vote against holding the defendant liable. However, this is not what happens when the group thought process that Pettit describes occurs. When group reasoning focuses on premises (individual judgment on each criterion), the judges render a different verdict: a two to one vote in favor of a liability verdict. This provides a different result, one that links the reasoning processes of the judges together in a way that is different from allowing the judges to exercise their judgments independently. If the three rocks behaved in this manner, then the weight would not be three pounds.

From Pettit's account, we can see how group reasoning can produce different results than members reasoning alone. It is important to note that the group reasoning process can actually be much more fluid and unstructured than the one Pettit provides. Group reasoning can be as simple as members reasoning individually and providing final judgments on an issue (one person/one vote). It can also involve members entering a group discussion with no thoughts on a subject and reasoning through the issue together to come to a truly joint decision. Or, a select number of members, or even just one member, can possess the authority to make decisions for the group. These select groups or individuals serve as the mind of the group. Whatever method is employed, it is clear that group discussions and member input can produce decisions and beliefs that no one had considered prior to the interaction and that differ from the decisions and beliefs of the individual members. Members consider institutional values, goals, and beliefs when making decisions; this would make their decisions radically different than decisions made from an individual perspective.

For a collection of individuals to be a group, its members must be committed to the group, the group must possess unity, and a group consciousness must exist. When these conditions are met, social order emerges. Olsen (1968) provides a good idea of what this entails when he writes that:

Social order emerges as ongoing relationships become interwoven into relatively stable and hence predictable arrangements. Stated differently, social order arises as the interactions of social actors become recurrent through time and complexly patterned. (p. 41).

This social order leads to the formation of a social group with properties that allow it to exercise non-redundant causal control over its members. ${ }^{4}$

\section{The Causal Powers of Groups}

We refer to and treat groups as if they possessed the sort of causal power that humans possess. In our daily discourse we typically refer to a group as an "it," and a group action or decision is often explained by claiming that a group performed that action or made this decision. We do not have to specify individual member names, but instead use the group name. In talking about groups in this manner, we are assigning agency to the group. The fact that we can do this, and that it is intelligible to others, gives some credence to the view that groups are objects. Sheehy (2006) argues this point when he states: "reference to groups is ineliminable or indispensable from our description and explanations of the social world" (p. 73) Many of our views on the social world would not make sense without this view of groups. However, the fact that we talk about and view groups in this way could be mistaken. So, it is necessary to address the primary objection that a conception of groups as objects will face.

To effectively argue that groups are objects, I will have to address the likely eliminativist objection that group actions and beliefs supervene on what its members do and believe. That is, I must consider the concern that group actions and beliefs could be produced by humans arranged groupwise. In what follows, I will present four cases that are supportive of the view that group actions and beliefs do not supervene on the actions and beliefs of its members.

\subsection{Co-location}

\footnotetext{
3 I thank Trenton Merricks for pressing me to address this objection.

4 Searle suggests that this "capacity for social cooperation is a biologically based capacity shared by humans and many other species." Our nature seems to be such that we are drawn to these social situations. John Searle (2003, p. 198), Social Ontology and Political Power, in Socializing Metaphysics: The Nature of Social Reality. Frederick F. Schmitt (Ed.) New York: Rowman and Littlefield Publishers.
} 
Imagine that a group of ten people is in a room. These ten people are members of a small business and make decisions about things such as the business' product lines, the business' core values, and the guidance given to the business' employees. However, these same ten people are also members of a reading group. As members of the reading group, they make decisions about things such as the books that members will read, how discussions will be conducted, and the location of meetings. If this were the case, the groups would be co-located. The ten people in the room would constitute both a small business and a reading group. ${ }^{5}$

The small business group and the reading group are composed of the same ten members; the two groups are co-located. Given this, it cannot be the case that the groups' properties supervene on their parts (the members). If group properties did supervene, the groups would be exactly the same. The small business would possess the same properties as the reading group. But this is not the case. The groups are distinct from one another. They perform different functions, have different causal power over the group's members, and within the two groups the members have different types of relationships. ${ }^{6}$ Consequently, because the groups are co-located and have non-supervenient properties, we can say that they possess non-redundant causal powers. The groups are objects.

A possible objection might be that the groups are not co-located because they have different functions. The members cannot be fulfilling the role of business and reading club members at the same time. While there may be some truth to this, it does not affect supervenience. If the groups' properties supervene on the members, they do so no matter what the members are doing. Supervenience will not vary according to what the members are discussing or the capacity in which they are acting. Because the groups are different, it cannot be said the properties of the group supervene on the 10 members. So, it is possible to attribute non-redundant causal power to the groups. Assuming that they meet the conditions that I specified in section three, the groups can be thought of as objects.

\subsection{Differing Beliefs}

Is it plausible to say that a group possesses different beliefs that its members? ${ }^{7}$ If it is, we can again say that a group's properties do not supervene on its parts, meaning that groups have non-redundant causal powers. There are two cases that illustrate how groups can have different beliefs than their members. In the first case, a group has a leader who has been given the authority by the group's members to speak and make decisions for the group as well as establish the group's beliefs. The group leader decides that the group, let's call it the End Timers, believes that the world will end on May 15, 2014. Once this decision is made, the belief is officially attributed to the group. When approached on the street, a man asks a group member the following question: "you are an End Timer, when do you think the world will end?" The End Timer responds: "we think the world will end on May 15, 2014." However, unbeknownst to the person asking the question, the End Timer membership (all group members but the leader) disagrees with the leader. They believe that the world will not end until June 1, 2014. Despite this fact, it can be fairly said that the group believes that the world will end on May 15, 2014, and that its members, acting in the capacity of group members, will reply with this date when asked when the group thinks the world will end. In this case the group membership, except for the leader, possesses different beliefs than the group.

An objection to this line of thought might be that it is not that the group has different beliefs than its members. What is happening is that the group members have two sets of beliefs: those as individuals and those as group members. Individually they believe in the later end of the world date, but as group members they stick to the party line. Since the leader has decided on a date, they are willing to go along with it. A problem with this objection is that the group members may only consider the issue as a group member; they may not even consider it in their life apart from the group. Thus, although they will disagree with the May 15, 2014 date only in their roles as group members, they can still coherently state the group's beliefs. That this is possible shows that groups can have different beliefs than their members. However, even if this is not the case, it is still possible to see the causal power of groups and their separateness from their members. Despite the fact that they do not agree with the group belief, group members are likely, when asked, to identify the group's belief as the world ending in 2014. Here, the group's belief is not simply a sum of the member's beliefs, but represents the belief of a separate entity. When in private, group members may admit that most End Timers believe differently, but will go on to say that the "group's belief is that the end will come on May 15, 2014." The ability to refer to the group separately from the member's beliefs is support for the view that groups are objects. They either believe that drug use is a fundamental right or is immoral.

A second case where group beliefs differ from those of its members involves compromise. Imagine a group of people

\footnotetext{
5 I thank Charlie Tanksley for suggesting this type of example during my presentation.

${ }^{6}$ Regarding the relationships, it might be the case that in the business two people are the chief executive officer and chief financial officer, while in the reading group they do not hold leadership positions but are just members.

${ }^{7}$ I thank Trenton Merricks for suggesting that this type of case needs to be addressed.
} 
founding a new country and creating a constitution. One half of the group believes that drug use should be illegal, while the other half believes that it should be legal. Both sides want a law to either prohibit drug use, because they consider it immoral, or to allow drug use, in order to protect a fundamental right. This issue threatens the development of the new constitution, so someone proposes a compromise. The compromise is accepted, and the group proposes that the issue of drug use is one that should be dealt with by the individual states rather than the central government. This is incorporated into the constitution and becomes law. The group's position and belief is that the legality of drug use should be determined by the individual states; however, none of the group members believe this. The group's belief differs from the beliefs of all its members.

It may be argued that what has happened here is that the group members adopted a new belief (that compromise was necessary to move forward) which eliminated the beliefs about drug legalization. Thus, if what we think of as groups are people arranged groupwise, the group members adopted the compromise belief and discarded the belief about drug legalization. A better explanation, however, seems to be that the members just possess different beliefs from their group. Even as individuals we often have conflicting beliefs, such as the belief that eating a second doughnut would be good versus the belief that a healthy diet is good. We may resist the belief that would prompt eating another doughnut; however, it is still a belief. It does not go away. So, while the person may resist the second doughnut based on the belief that a healthy diet is good, we can still say that the person possesses both beliefs. In a similar sense, we would say that the group members' belief differs from the belief of the group. The group creating the constitution believes that the drug legalization issue was best left to the states, while the group's members disagree because they possess strong beliefs on both sides of the issue.

\subsection{Loyalty to Groups}

Members of groups feel an affiliation and identity with other group members, and these bonds are an important part of a cohesive group. However, members also possess bonds of loyalty to the group as an institution, and these bonds are often as strong as or stronger than the bonds with other members. This can create a difficult decision for a member who feels compelled, due to the group's standards, to report other members for wrongdoing. In many cases the individual does not want to do this because he feels disloyal to his comrades; however, the group, through its norms, beliefs, and values, causes him to act. This pressure from the group causes him to report his peers, even though other members of the group oppose this action and try to persuade the person not to report the wrongdoing. The group's pressure on the individual cannot be explained in terms of the actions of other members or by considering a group to be people arranged groupwise. The fact that individuals can be loyal to a group's institutional values and ideals, and not merely to the values and ideals of its members, suggests that the group is a separate entity and possesses non-redundant causal properties.

What is lost if people are loyal to people arranged groupwise rather than to a group ${ }^{8}$ I think there is a distinct difference between the two loyalties. Loyalty to people arranged groupwise suggests loyalty to individuals. It is to be loyal merely to the group's members or to the sum of their beliefs and values. Loyalty to people arranged groupwise would not seem to survive a complete change of membership. By acknowledging the existence of a group, one's loyalty transcends mere loyalty to one's fellow group members. It can survive complete member replacement as well as a disagreement with fellow members.

Even if people arranged groupwise could survive total replacement of members and disagreement within the ranks, this account of groups does not explain many of the effects that groups have. Group members give up agency in certain areas when joining a group. This is because the group possesses a consciousness that is separate and distinct from that of its members. This consciousness exercises downward causation on the members by affecting their beliefs and actions. Groups constrain and guide the behavior of their members and impart beliefs upon them as well. Members are changed by participation in groups because of this downward causation. One cannot be loyal to a group without recognizing these facts, because loyalty seems to require that one allow herself to be influenced by the group. Accepting this influence is accepting the non-redundant causal power of the group.

\section{Conclusion}

Social groups cause things that cannot be explained by mere reference to their members. Social groups have consciousness and beliefs that can be clearly different from their members' consciousness and beliefs. To deny the existence of social groups in light of this fact would be like admitting that there is group consciousness and reasoning process while denying the group that possesses these attributes. Social group members are held collectively responsible for the group's actions (a sign of agency), even when it is clear that a majority of the group members did not perform the action. Social groups possess a history and reputation that is not connected in any way to its current membership;

\footnotetext{
${ }^{8}$ I thank Trenton Merricks for posing this question during my presentation.
} 
however, this reputation sticks to current group members, despite the fact that they have had no part in achieving it. These facts, and the ones mentioned above, illustrate that what social groups cause and believe cannot be attributed to supervenience on its members' actions and beliefs. Member interaction creates a social order which is instantiated in a stable and unified organization with committed members. This, in turn, makes a social group an object.

\section{References}

Gilbert, M. (1989). On Social Facts. New York: Routledge.

Gilbert, M. (2003). The Structure of the Social Atom: Joint Commitment as the Foundation of Human Social Behavior. In Frederick F. Schmitt (Ed.), Socializing Metaphysics: The Nature of Social Reality, 39-64. New York: Rowman and Littlefield Publishers.

Merricks, Trenton (2003). Objects and Persons. Oxford: Clarendon Press.

Olsen, M. E. (1968). The Process of Social Organization. New York: Holt, Rinehart and Winston.

Pettit, P. (2003). Groups with Minds of Their Own. In Socializing Metaphysics: The Nature of Social Reality, In Frederick F. Schmitt (Ed.), Socializing Metaphysics: The Nature of Social Reality, 167-194. New York: Rowman and Littlefield Publishers.

Searle, J. (2003). Social Ontology and Political Power. In Frederick F. Schmitt (Ed.), Socializing Metaphysics: The Nature of Social Reality, 195-210. New York: Rowman and Littlefield Publishers.

Sheehy, P. (2006). The Reality of Social Groups. Hampshire: Ashgate Publishing.

\section{$(\mathrm{cc}) \mathrm{BY}$}

This work is licensed under a Creative Commons Attribution 3.0 License. 